\title{
TITLE:
}

\section{Quantum Variational Principle of Irreversible Processes in a Magnetic field}

\author{
$\operatorname{AUTHOR}(\mathrm{S})$ : \\ Hattori, Masumi; Nakano, Huzio
}

\section{CITATION:}

Hattori, Masumi ... [et al]. Quantum Variational Principle of Irreversible Processes in a Magnetic field. 数理解析研究所講究録 1997, 982: 79-89

\section{ISSUE DATE:}

1997-03

URL:

http://hdl.handle.net/2433/60919

RIGHT: 


\title{
Quantum Variational Principle of Irreversible Processes in a Magnetic field
}

\author{
服部蕒澄 (Masumi Hattori) \\ Department of Physics, Nagoya Institute of Technology, \\ Nagoya 466, Japan \\ 中野藤生 (Huzio Nakano) \\ 3-110, Issiki-sinmati 2-401, Nakagawa-ku, Nagoya 454, Japan
}

\begin{abstract}
Contractions of the density matrix in a magnetic field, are performed with no approximation in two steps: the off-diagonal part of the density matrix is eliminated first and then the even part with respect to time reversal. In consequence the maximum variational principle in connection with the entropy production is derived for the odd part of diagonal elements of the density matrix, which means the contraction of the density matrix brings about irreversibility.
\end{abstract}




\section{Introduction}

Since one of the present authors (H.N.) formulated a quantum variational principle for the von Neumann equation[1], in contrast to the classical one for the conventional Boltzmann-Bloch equation [2], we have paid attention to the fact that the information contraction in the variational principle brings about irreversibility $[1,3,4,5]$. In a previous paper[1, 3, 5], we have derived the maximum variational principle in the absence of a magnetic field, by contracting the density matrix with no approximation.

In this paper, we generalize the investigation to the case of presence of a magnetic field. In Sec.2, the stationarity variational principle is presented for the von Neumann equation. We first perform a contraction in Sec.3 to eliminate the off-diagonal part of the density matrix, in the scheme of Stinchcombe's non-orthogonal representation [6], where we can perform the similar contraction as in the absence of a magnetic field. Then we get a variational principle for the diagonal part, which still remains to be a stationarity problem. In Sec.4, we secondly perform another contraction to eliminate the even part with respect to time reversal. We then attain to a maximum principle for the odd part of diagonal elements of the density matrix. We finally summarize the results in Sec.5. 


\section{General formulation}

The Hamiltonian of the system in a magnetic field $\mathbf{H}$ is expressed as

$$
H_{t o t}=\sum_{\mathbf{k}, \mathbf{k}^{\prime}}\left\langle\mathbf{k}|h| \mathbf{k}^{\prime}\right\rangle a_{\mathbf{k}}^{\dagger} a_{\mathbf{k}^{\prime}}
$$

in terms of the creation operator $a_{\mathbf{k}}^{\dagger}$ and annihilation operator $a_{\mathbf{k}}$, respectively, of the conduction electron with wave number $\mathbf{k}$, where

$$
h \equiv h_{0}+V
$$

is composed of the kinetic energy

$$
h_{0} \equiv \frac{1}{2} m \mathbf{v}^{2}
$$

due to the velocity

$$
\mathbf{v} \equiv \frac{1}{m}\left(\mathbf{p}-\frac{e}{c} \mathbf{A}\right)
$$

and scattering potential $V$ by impurities. The vector potential is gauged as

$$
\mathbf{A}=(0, H x, 0)
$$

The density matrix $\rho$ is expressed as

$$
\rho(t)=\rho_{c}+\int_{0}^{\beta} d \lambda \rho_{c} \exp \left(\lambda H_{t o t}\right) \Phi(t) \exp \left(-\lambda H_{t o t}\right),
$$

where

$$
\begin{aligned}
\rho_{c} & \equiv K \exp \left[\beta\left(H_{t o t}-\mu N\right)\right] \\
\Phi(t) & \equiv \sum_{\mathbf{k}, \mathbf{k}^{\prime}}\left\langle\mathbf{k}|\phi(t)| \mathbf{k}^{\prime}\right\rangle a_{\mathbf{k}}^{\dagger} a_{\mathbf{k}^{\prime}}
\end{aligned}
$$


The von Neumann equation in an external electric field $\mathbf{E}(t)$ is

$$
\frac{\partial \phi(t)}{\partial t}+l \phi(t)=e \mathbf{v} \cdot \mathbf{E}(t)
$$

where the superoperator $l$ acting on the state operator $\phi$ is defined as

$$
l \phi \equiv \frac{i}{\hbar}[h, \phi]
$$

which is divided into two parts as

$$
\begin{aligned}
& l=l_{0}+\delta l, \\
& l_{0} \phi \equiv \frac{i}{\hbar}\left[h_{0}, \phi\right], \\
& \delta l \phi \equiv \frac{i}{\hbar}[V, \phi] .
\end{aligned}
$$

We consider situations in duality according to the time boundary conditions:

$$
\begin{array}{ll}
\mathbf{E}(t)=\mathbf{E} \exp (s t) & (t<0), \quad \phi(t)=\phi^{(+)} \exp (s t) \\
\mathbf{E}(t)=\mathbf{E} \exp (-s t) & (t>0), \quad \phi(t)=\phi^{(-)} \exp (-s t)
\end{array}
$$

similarly to the situations of incoming and outgoing waves in scattering theory [7], where $\mathbf{E}$ is a constant electric field and $s$ is a infinitesimal. The von Neumann equations $(2.9)$ is rewritten

$$
\begin{aligned}
(s+l) \phi^{(+)} & =e \mathbf{v} \cdot \mathbf{E} \\
(-s+l) \phi^{(-)} & =e \mathbf{v} \cdot \mathbf{E}
\end{aligned}
$$


for $\phi^{(+)}$and $\phi^{(-)}$, respectively. Using the Fermi distribution function

$$
f \equiv \frac{1}{\exp (\beta h-\beta \mu)+1},
$$

we define the inner product between $\phi$ and $\psi$ as

$$
\langle\phi, \psi\rangle=\langle\psi, \phi\rangle \equiv \int_{0}^{\beta} d \lambda \operatorname{tr}[\phi f \exp (\lambda h) \psi \exp (-\lambda h)(1-f)] .
$$

The average electric current for the present is obtained as

$$
\mathbf{J}=\left\langle e \mathbf{v}, \phi^{(+)}\right\rangle=-\left\langle e \mathbf{v}, \phi^{(-)}\right\rangle
$$

Now, making a variational functional

$$
W\left(\phi^{(+)}, \phi^{(-)}\right) \equiv\left\langle\phi^{(-)},(s+l) \phi^{(+)}\right\rangle+\left\langle\phi^{(+)}-\phi^{(-)}, e \mathbf{v} \cdot \mathbf{E}\right\rangle
$$

stationary as to $\phi^{(+)}$and $\phi^{(-)}$, we get the von Neumann equations (2.16) and (2.17), where the stationary value gives the average current $(2.20)$.

\section{Elimination of off-diagonal parts}

The velocity $\mathbf{v}$ is off-diagonal in the scheme of diagonalizing the unperturbed Hamiltonian $h_{0}$, in contradistinction to the case of no magnetic field. Therefore, this scheme is inadequate to carrying out the contraction and we instead adopt a non-orthogonal representation proposed by Stinchcombe[6], where the velocity is diagonal and the contraction can be performed in the same way as in the case of no magnetic field. The $\mathbf{k}, \mathbf{k}^{\prime}$ element of any operator $\phi$ is defined as

$$
\left\{\mathbf{k}|\phi| \mathbf{k}^{\prime}\right\} \equiv \int d \mathbf{r}_{1} d \mathbf{r}_{2} \exp \left(-i \epsilon X_{12} y_{12}\right)\left\langle\mathbf{k} \mid \mathbf{r}_{1}\right\rangle\left\langle\mathbf{r}_{1}|\phi| \mathbf{r}_{2}\right\rangle\left\langle\mathbf{r}_{2} \mid \mathbf{k}^{\prime}\right\rangle
$$


where the triangular brackets denote the usual orthogonal representation and

$$
\begin{aligned}
\epsilon & \equiv \frac{e H}{\hbar c}, \\
X_{12} & \equiv \frac{1}{2}\left(x_{1}+x_{2}\right), \quad y_{12} \equiv y_{1}-y_{2} .
\end{aligned}
$$

Let us decompose $\phi^{( \pm)}$into the diagonal part $\phi_{d}^{( \pm)}$and the off-diagonal part $\phi_{o d}^{( \pm)}$in Stinchcombe's representation as

$$
\begin{aligned}
& \phi^{(+)}=\phi_{d}^{(+)}+\phi_{o d}^{(+)} \\
& \phi^{(-)}=\phi_{d}^{(-)}+\phi_{o d}^{(-)} .
\end{aligned}
$$

We introduce the projection superoperator $P$ which projects any operator onto the diagonal operator and the complementary $Q=1-P$. Applying $Q$ to both sides of the von Neumann equation (2.16), we get

$$
Q(s+l) \phi^{(+)}=0
$$

By substituting (3.4) for $\phi^{(+)}$into (3.6), we obtain

$$
\phi_{o d}^{(+)}=-[Q(s+l) Q]^{-1} Q \delta l P \phi_{d}^{(+)} .
$$

Substituting (3.7) into (3.4), we can write

$$
\begin{aligned}
& \phi^{(+)}=a_{s} \phi_{d}^{(+)} \\
& (s+l) \phi^{(+)}=(\tilde{l}+\tilde{m}) \phi_{d}^{(+)},
\end{aligned}
$$

where we have defined

$$
a_{s} \equiv 1-[Q(s+l) Q]^{-1} Q \delta l P,
$$




$$
\begin{aligned}
& \tilde{l} \equiv s P-\frac{1}{2} P \delta l Q\left([Q(s+l) Q]^{-1}-[Q(-s+l) Q]^{-1}\right) Q \delta l P \\
& \tilde{m} \equiv P l P-\frac{1}{2} P \delta l Q\left([Q(s+l) Q]^{-1}+[Q(-s+l) Q]^{-1}\right) Q \delta l P
\end{aligned}
$$

Changing the sign of $s$ in (3.7), (3.8) and (3.9), we get

$$
\begin{aligned}
& \phi_{o d}^{(-)}=-[Q(-s+l) Q]^{-1} Q \delta l P \phi_{d}^{(-)} \\
& \phi^{(-)}=a_{-s} \phi_{d}^{(-)} \\
& (-s+l) \phi^{(-)}=(-\tilde{l}+\tilde{m}) \phi_{d}^{(-)}
\end{aligned}
$$

respectively. Substituting (3.8), (3.9) and (3.14), we can rewrite (2.21) as

$$
\begin{aligned}
& W\left(a_{s} \phi_{d}^{(+)}, a_{-s} \phi_{d}^{(-)}\right) \\
& =\left\langle a_{-s} \phi_{d}^{(-)},(\tilde{l}+\tilde{m}) \phi_{d}^{(+)}\right\rangle+\left\langle a_{s} \phi_{d}^{(+)}-a_{-s} \phi_{d}^{(-)}, e \mathbf{v} \cdot \mathbf{E}\right\rangle .
\end{aligned}
$$

By making stationary of (3.16), we get the master equations

$$
\begin{aligned}
(\tilde{l}+\tilde{m}) \phi_{d}^{(+)} & =e \mathbf{v} \cdot \mathbf{E} \\
(-\tilde{l}+\tilde{m}) \phi_{d}^{(-)} & =e \mathbf{v} \cdot \mathbf{E} .
\end{aligned}
$$

We can show that these master equations have the form of the conventional Boltzmann-Bloch equation.

\section{Elimination of even part as to time rever- sal}

Let us define the time reversal of an operator $\alpha(\mathbf{H})$ depending on the magnetic field $\mathbf{H}$ as $\overline{\alpha(\mathbf{H})}$. Here we mean the time reversal for inverting the 
magnetic field $\mathbf{H}$ as well as the time; by applying the time reversal to the external system generating the magnetic field as well as the system. Thus,

$$
\left\langle\mathbf{r}|\overline{\alpha(\mathbf{H})}| \mathbf{r}^{\prime}\right\rangle \equiv\left\langle\mathbf{r}^{\prime}|\alpha(-\mathbf{H})| \mathbf{r}\right\rangle
$$

in the representation scheme of diagonalizing the spatial coordinate.

Applying the time reversal to the von Neumann equation (2.16), we get

$$
\left(s-l_{\mathbf{H}}\right) \overline{\phi(+)(\mathbf{H})}=-e \mathbf{v}(\mathbf{H}) \cdot \mathbf{E} .
$$

where the dependence of the superoperator $l$ on a magnetic field has been explicitly shown as $l_{\mathbf{H}}$. Comparing (4.2) with (2.17) we can write

$$
\phi^{(-)}(\mathbf{H})=\overline{\phi^{(+)}(\mathbf{H})}
$$

whence we decompose $\phi_{d}^{(+)}$and consequently $\phi_{d}^{(-)}$as

$$
\begin{aligned}
& \phi_{d}^{(+)}(\mathbf{H})=\phi_{d}^{\prime}(\mathbf{H})+\phi^{\prime \prime}{ }_{d}(\mathbf{H}), \\
& \phi_{d}^{(-)}(\mathbf{H})=-\phi_{d}^{\prime}(\mathbf{H})+\phi_{d}(\mathbf{H}),
\end{aligned}
$$

into $\phi_{d}^{\prime}$ and $\phi^{\prime \prime}{ }_{d}$ which denote the odd and even parts as to time reversal, respectively. Omitting the argument $\mathbf{H}$, we can rewrite (3.16)

$$
\begin{aligned}
& W\left(a_{s}\left(\phi_{d}^{\prime}+\phi_{d}{ }_{d}\right), a_{-s}\left(-\phi_{d}^{\prime}+\phi_{d}\right)\right)=\left\langle a_{-s}\left(-\phi_{d}^{\prime}+\phi_{d}{ }_{d}\right),(\tilde{l}+\tilde{m})\left(\phi_{d}^{\prime}+\phi_{d}\right)\right\rangle \\
& +\left\langle\left(a_{s}+a_{-s}\right) \phi_{d}^{\prime}+\left(a_{s}-a_{-s}\right) \phi_{d}, e \mathbf{v} \cdot \mathbf{E}\right\rangle
\end{aligned}
$$

as a functional of diagonal parts $\phi_{d}^{\prime}$ and $\phi "{ }_{d}$, which is made stationary by

$$
\begin{aligned}
& (\tilde{l}+\tilde{m})\left(\phi_{d}^{\prime}+\phi_{d}{ }_{d}\right)+\left(-\tilde{l}_{s}+\tilde{m}\right)\left(-\phi_{d}^{\prime}+\phi^{\prime \prime}{ }_{d}\right)=2 e \mathbf{v} \cdot \mathbf{E}, \\
& (\tilde{l}+\tilde{m})\left(\phi_{d}^{\prime}+\phi_{d}{ }_{d}\right)=(-\tilde{l}+\tilde{m})\left(-\phi_{d}^{\prime}+\phi^{\prime \prime}{ }_{d}\right)
\end{aligned}
$$


latter of which is solved as

$$
\phi_{d}{ }_{d}=-[\tilde{l}]^{-1} \tilde{m} \phi_{d}^{\prime}
$$

Substituting (4.9) into (3.8), (3.9), (3.14)and (3.15), we obtain

$$
\begin{aligned}
& \phi^{(+)}=a_{s}\left(1-\left[\tilde{l}^{-1} \tilde{m}\right) \phi_{d}^{\prime}\right. \\
& \phi^{(-)}=-a_{-s}\left(1+\left[\tilde{l}^{-1} \tilde{m}\right) \phi_{d}^{\prime}\right. \\
& (s+l) \phi^{(+)}=(-s+l) \phi^{(-)}=\left(\tilde{l}_{s}-\tilde{m}[\tilde{l}]^{-1} \tilde{m}\right) \phi_{d}^{\prime}
\end{aligned}
$$

Inserting (4.9) and (4.12) in (4.6), we get the maximum principle

$$
\begin{aligned}
& W\left(a_{s}\left(1-[\tilde{l}]^{-1} \tilde{m}\right) \phi_{d}^{\prime},-a_{-s}\left(1+[\tilde{l}]^{-1} \tilde{m}\right) \phi_{d}^{\prime}\right) \\
& =\left\langle-a_{-s}\left(1+[\tilde{l}]^{-1} \tilde{m}\right) \phi_{d}^{\prime},\left(\tilde{l}-\tilde{m}[\tilde{l}]^{-1} \tilde{m}\right) \phi_{d}^{\prime}\right\rangle \\
& +\left\langle\left(\left(a_{s}+a_{-s}\right)-\left(a_{s}-a_{-s}\right)[\tilde{l}]^{-1} \tilde{m}\right) \phi_{d}^{\prime}, e \mathbf{v} \cdot \mathbf{E}\right\rangle
\end{aligned}
$$

which should be maximized as a functional of $\phi_{d}^{\prime}$. Maximizing (4.13) leads to a master equation

$$
\left(\tilde{l}-\tilde{m}\left[\tilde{l}^{-1} \tilde{m}\right) \phi_{d}^{\prime}=e \mathbf{v} \cdot \mathbf{E}\right.
$$

where the maximum is equal to

$$
W_{\max }=\left\langle a_{s}\left(1-[\tilde{l}]^{-1} \tilde{m}\right) \phi_{d}^{\prime}, e \mathbf{v} \cdot \mathbf{E}\right\rangle=\left\langle a_{-s}\left(1+[\tilde{l}]^{-1} \tilde{m}\right) \phi_{d}^{\prime}, e \mathbf{v} \cdot \mathbf{E}\right\rangle=\mathbf{J} \cdot \mathbf{E}
$$

The present variational principle is formally quite similar to that derived from the Boltzmann-Bloch equation by Bailyn [8], who derived the maximum variational principle for the even part with respect to a magnetic field, which corresponds to the odd part $\phi_{d}^{\prime}$. 


\section{Conclusion}

Contracting the density matrix, we have converted the stationarity variational principle for the density matrix in a magnetic field, into the maximum principle for the diagonal elements of the odd component of the density matrix with respect to time reversal in the scheme of Stinchcombe's representation, with no perturbation. We can see the features of the variational principles in the course of contractions in Table below.

Table. Variational principles in the whole course of contractions.

\begin{tabular}{|l|l|l|l|}
\hline Character & stationarity & stationarity & maximum \\
\hline Variables & $\phi^{(+)}, \phi^{(-)}$ & $\phi_{d}^{(+)}, \phi_{d}^{(-)}$ & $\phi_{d}^{\prime}$ \\
\hline Euler & von Neumann & master equations & master equation \\
equations & equations & $(3.17)$ and (3.18) & $(4.14)$ \\
& $(2.16)$ and $(2.17)$ & which are equivalent & which is equivalent \\
& & to the rectified & to the rectified \\
& & Boltzmann-Bloch & Boltzmann-Bloch \\
& & equations & equation \\
\hline
\end{tabular}

The maximum principle is formally the same as that for the conventional Boltzmann-Bloch equation in a magnetic field and related to the entropy production characteristic of irreversible process. In this sence, we could show that the contractions of the density matrix bring about irreversibility also in a magnetic field. 


\section{References}

[1] H.Nakano, Proc.Phys.Soc.82, 757(1963).

[2] K.Umeda, Sci.Pap.Inst.Phys. Chem. Res.Tokyo,39,342(1942).

M.Kohler, Z.Phys.124,772(1948);125,679(1949).

E.H.Sondheimer, Proc.Phys.Soc.A203,75(1950).

A.H.Wilson, The Theory of Metals (Cambridge Univ.Press,1953).

J.M.Ziman, Electrons and Phonons (Clarendon press,Oxford,1962).

[3] H.Nakano and M.Hattori, Prog.Theor.Phys.83,1115(1990).

[4] H.Nakano, Int.J.Mod.Phys.B7,2397(1993), and references therein.

[5] M.Hattori and H.Nakano, Int.J.Mod.Phys.B9,1227(1995).

[6] R.B.Stinchcombe, Proc.Phys.Soc.78,275(1961).

[7] B.A.Lippmann and J.Schwinger, Phys.Rev.79,469(1950).

[8] M.Bailyn, Phys.Rev.126,2040(1962), and references therein. 